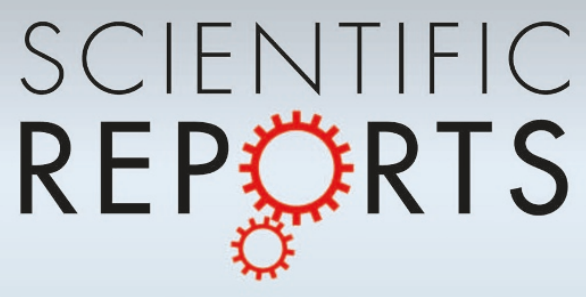
Controlled Deformation of a PDMS

SUBJECT AREAS: NANOBIOTECHNOLOGY

NANOFLUIDICS

BIOPHYSICS

APPLIED PHYSICS

Received

25 July 2012

Accepted

28 September 2012

Published

9 November 2012

Correspondence and requests for materials should be addressed to

P.F. (paola.fanzio@ unige.it)

* These two authors contributed equally to this work.

\section{Nanochannel Device}

\author{
Paola Fanzio ${ }^{*}$, Chiara Manneschi ${ }^{1 *}$, Elena Angeli ${ }^{1}$, Valentina Mussi' , Giuseppe Firpo' , Luca Ceseracciu2 \\ Luca Repetto' \& Ugo Valbusa'
}

${ }^{1}$ Nanomed Labs, Dipartimento di Fisica, Università di Genova, via Dodecaneso 33 Genova, 16146, Italy, ${ }^{2}$ Nanophysics, Istituto Italiano di Tecnologia (IIT), via Morego, 30 Genova, 16163, Italy.

Several strategies have been developed for the control of DNA translocation in nanopores and nanochannels. However, the possibility to reduce the molecule speed is still challenging for applications in the field of single molecule analysis, such as ultra-rapid sequencing. This paper demonstrates the possibility to alter the DNA translocation process through an elastomeric nanochannel device by dynamically changing its cross section. More in detail, nanochannel deformation is induced by a macroscopic mechanical compression of the polymeric device. This nanochannel squeezing allows slowing down the DNA molecule passage inside it. This simple and low cost method is based on the exploitation of the elastomeric nature of the device, can be coupled with different sensing techniques, is applicable in many research fields, such as DNA detection and manipulation, and is promising for further development in sequencing technology.

$\mathrm{N}$ anofluidics is well established as a tool for the study and the development of new methods in single molecule analysis. In particular, nanopores and nanochannels are used for many applications, such as biomolecule separation, detection and manipulation ${ }^{1}$.

In this context, the slowing down and the control of the conformation of single DNA molecules inside nanoconfining structures are critical issues. As an example, a challenging application such as ultra-fast DNA sequencing would benefit from an accurate control of single molecule dynamics. The working principle is based on the analysis of ionic current fluctuations elicited by the electrophoretic passage of DNA molecules inside a single nanochannel or nanopore. In principle, single nucleotide detection can be achieved by discriminating their different current signature ${ }^{2}$. Nevertheless, several problems remain to be solved. In fact, the high speed of DNA strand translocation (about $5 \mu$ s per base) does not allow to accurately measure and discriminate ionic current fluctuations for the four distinct DNA bases. Indeed, such a sensitive measurement of DNA translocation signature would require an extremely high bandwidth, with the drawback of increasing baseline recording noise up to levels higher than single base current fluctuations, thus preventing their discrimination.

Furthermore, the slowing down and the manipulation of DNA strands ${ }^{3}$ would significantly improve the sensitivity of single molecule electrical detection in many other applications, such as the study of the unravelling ${ }^{4}$ and conformation ${ }^{5}$ of ssDNA and dsDNA inside nanopores or nanochannels, and the realization of force measurements on DNA molecules ${ }^{6}$.

The translocation speed of DNA can be reduced by lowering temperature ${ }^{7}$, increasing solvent viscosity, decreasing potential bias between the two sides of the pore or channel ${ }^{8-10}$ and modifying the surface charge ${ }^{11}$ (by changing molarity and $\mathrm{pH}$ of the solution). However, all these methods are often associated with high noise level, which prevents DNA detection. To overcome this limit, the spatial control of DNA inside the nanoconfinement region can be achieved by using optical tweezers ${ }^{6,12,13}$ or by labeling target molecules with particles or markers, such as magnetic beads ${ }^{14}$, which cause a reduction in the polymer speed without impairing ionic currents. However, a complex and often expensive preparation of the sample before the measurement is the main disadvantage of these techniques.

An alternative approach to alter translocation dynamics is based on the possibility to dynamically change the nanostructure size. Biological and solid state nanopores have a fixed dimension that cannot be tuned during measurements. Several strategies have been developed to overcome this limitation, all based on nanopore functionalization with biopolymers able to change their conformation depending on the external conditions, 


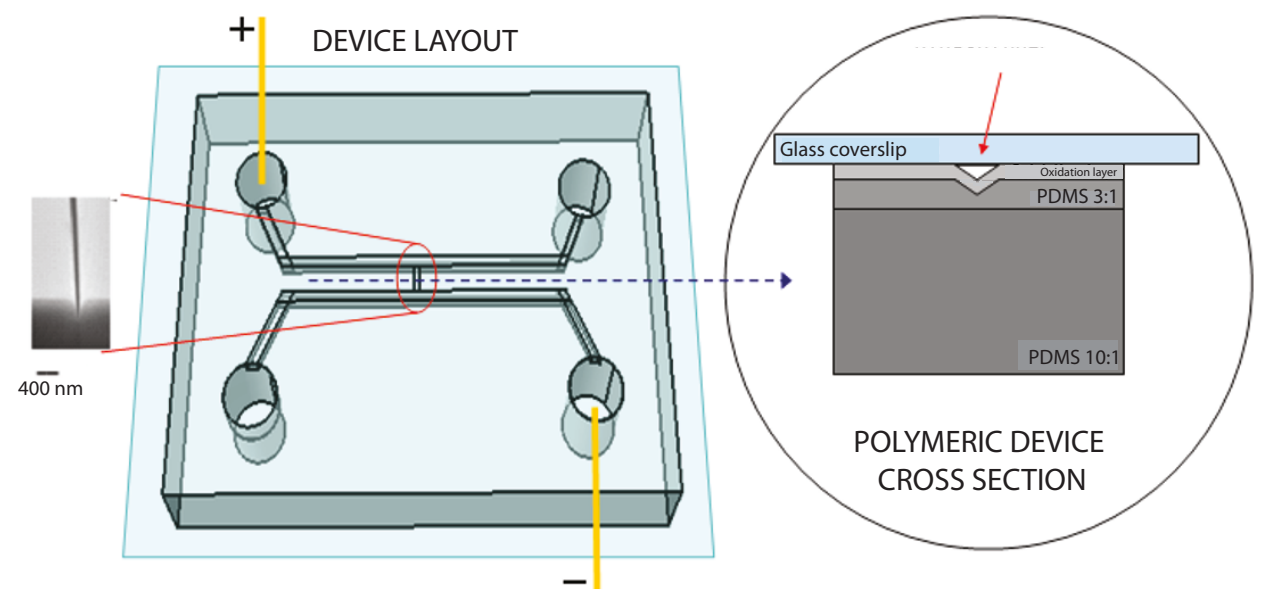

Figure $1 \mid$ Device layout. On the left, sketch of the top view of the device layout: two microchannels are connected by a single nanochannel (which is shown in the SEM image) and two electrodes (yellow) allow applying the voltage and collecting the ionic current. On the right, a cross section of the polymeric device in correspondence of the blue arrow (where the triangular nanochannel is present): a thick PDMS 10:1 layer supports a thin PDMS 3:1 layer which reproduces the nano and micro features. An oxygen plasma treatment causes the formation of an oxidation layer and allows bonding the polymer with a glass cover slip in order to close the fluidics.

such as voltage ${ }^{15}$ or temperature ${ }^{16}$. However, chemical activations are generally not well controlled ${ }^{17}$ and detection is influenced by interactions between target molecules and the functionalization layer.

The use of elastomeric materials opens up new possibilities to obtain resizable devices ${ }^{18,19}$. As an example, Sowerby et al. ${ }^{20,21}$ described the use of a single resizable nanopore that can immobilize and manipulate polynucleotides, thus prolonging their translocation time.

Here we propose a new method that exploits the elastomeric behavior of soft polymeric materials to fabricate tuneable devices for the manipulation and trapping of DNA molecules inside a nanochannel ${ }^{22,23}$. In particular, we present the fabrication process of an elastomeric Lab-On-Chip device and the characterization of the nanochannel resizing in response to a compressive strain. A DNA detection experiment is reported, showing that the molecular translocation time increases when the nanochannel cross section is decreased.

\section{Results}

Device fabrication and characterization. The device consists of a single nanochannel linking two microchannels, as shown in the top view sketch of figure 1 (DEVICE LAYOUT and SEM image). Four cylindrical reservoirs are present in order to connect the device to an external fluidic apparatus and to insert wire electrodes.

The fabrication procedure (see Methods section) is based on a double replica molding process: in particular, poly(dimethylsiloxane) (PDMS) is used to obtain a polymeric device starting from a micro- and nano-patterned silicon master ${ }^{24}$. Its properties, such as good biocompatibility, thermal stability, homogeneity, chemical inertness and durability ${ }^{25}$, make it suitable for our application.

The final device is a composite stamp made of three polymeric layers, as shown in the schematic cross section of figure 1 (POLYMERIC DEVICE CROSS SECTION): a thick PDMS 10:1 stab, a thin PDMS 3:1 layer and an oxidation layer. This last layer is produced by an $\mathrm{O}_{2}$ plasma treatment that is performed in order to close the device with a glass coverslip.

The thickness of the oxidation layer was measured by Atomic Force Microscopy (AFM) following the procedure outlined by Mills et al. ${ }^{26}$, finding a value of about $100 \mathrm{~nm}$. This value is comparable with the dimension of the nanochannel, which was also evaluated by AFM measurements on the PDMS positive replica. The height profile shows a $\mathrm{V}$-shaped cross-section with an estimated depth $\mathrm{h}=(70 \pm 5) \mathrm{nm}$ and $\mathrm{a}$ width $\mathrm{b}=(1.0 \pm 0.3) \mu \mathrm{m}$. The length of the nanochannel is $15 \mu \mathrm{m}$.
Uniaxial compressive tests gave a similar value for the Young's modulus of PDMS 10:1 and PDMS 3:1 layers, which is equal to about $2 \mathrm{MPa}$, for small deformations.

Squeezing experiment. The elastomeric nature of the material allowed changing the nanochannel cross section by imposing a compressive strain on the top of the device.

The polymeric nanochannel squeezing experiment was performed by anchoring the device to a rigid support and imposing a compression on the polymeric top of the device by means of a calibrated moving screw, as shown in figure $2 \mathrm{a}$. The displacement of the screw with respect to the initial position $(\Delta \mathrm{L})$ corresponds to the reduction of the thickness of the device with respect to its value when no compression is applied $\left(\mathrm{L}_{0}=5 \mathrm{~mm}\right)$. So, each value of compressive strain, $\mathrm{D}=\Delta \mathrm{L} / \mathrm{L}_{0}$, corresponds to a nanochannel shrinking.

After filling the device with $\mathrm{KCl} 1 \mathrm{M}$ solution, the ionic current was measured at different applied voltages by means of two $\mathrm{Ag} / \mathrm{AgCl}$ electrodes connected to a patch clamp amplifier.

The current/voltage curve was linear, and could be fitted to obtain the electrical conductance of the nanochannel, $\mathrm{G}^{27}$. Neglecting the surface conductance contribution ${ }^{28}$ and the access resistance ${ }^{29}$, the nanochannel section $S$ is related to its conductance, its length 1 , and the ionic conductivity of the solution $\sigma$, by the Ohm's relation:

$$
\mathrm{S}=1 \frac{\mathrm{G}}{\sigma}
$$

Since the channel conductance is directly related to its cross section, the nanochannel squeezing was measured by means of electrical recordings. Therefore, during the compression, the electrical response was monitored and the results are shown in figure $2 \mathrm{~b}$ (black squares), where the conductance (G) normalized to its value at $\Delta \mathrm{L}=$ $0\left(G_{0}\right)$ is plotted as a function of $D$. When the imposed displacement increases, the conductance decreases, due to the reduction of the cross section of the nanochannel. These measurements were repeated 10 times by using a different device each time, obtaining compatible results: if a device is correctly filled with the salt solution, the squeezing experiment can be performed and the result follows the trend shown in figure $2 \mathrm{~b}$. This means that the reproducibility of the experiment is mainly related to the fabrication process: about the $80 \%$ of devices can be effectively used for ionic current measurements.

Simulations of nanochannel deformation. Numerical simulations of the squeezing process were performed using Comsol Multiphysics 
a)

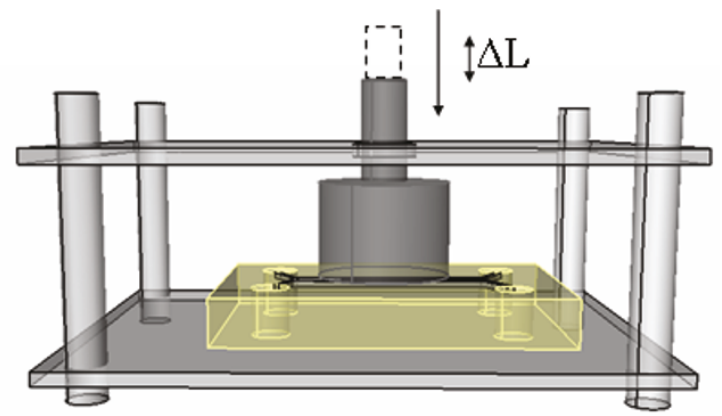

b)

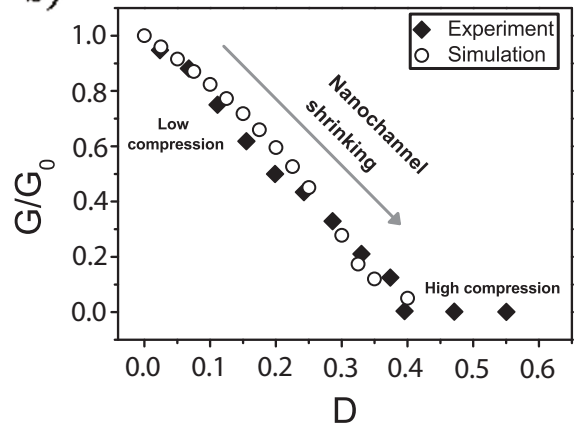

c)

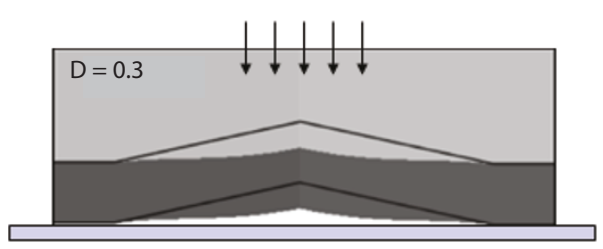

Nanochannel deformation

Figure $2 \mid$ Squeezing experiment. (a) Sketch of the squeezing setup (b) Conductance $\mathrm{G}$ normalized to its zero-displacement value $\mathrm{G}_{0}$ plotted versus compressive strain D, experimental data (squares) and simulations of the device (circles) (c) Simulated profile of the nanochannel for $\mathrm{D}=0.3$; the bulk PDMS layer and the oxidation layer domains are represented in light and dark grey, respectively. The not deformed channel shape is indicated by a black line.
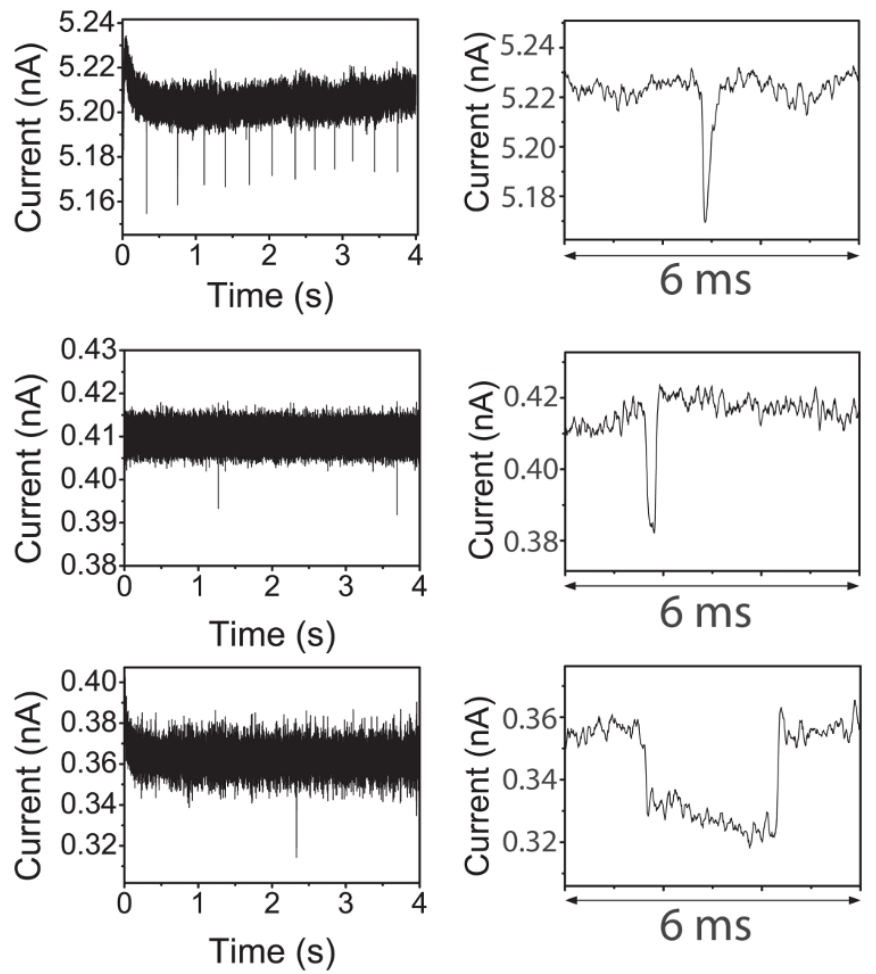

Figure 3 DNA detection. Current traces recorded after the insertion of a solution containing $\lambda$-DNA molecules for (a) $\mathrm{D}=0$, (b) $\mathrm{D}=0.3$ and (c) $\mathrm{D}$ $=0.35$. Magnification of a single translocation event for $(\mathrm{d}) \mathrm{D}=0,(\mathrm{e}) \mathrm{D}=$ 0.3 and (f) $\mathrm{D}=0.35$. All current traces were acquired applying a voltage bias of $200 \mathrm{mV}$. 3.5a. The device was modeled as a bilayer made of a bulk PDMS layer and an oxidation layer.

Results of the simulations are shown in figure $2 \mathrm{~b}$ (circles). The experimental squeezing behaviour (figure $2 \mathrm{~b}$, squares) is well described by the numerical simulations (figure $2 \mathrm{~b}$, circles) performed using $\mathrm{E}_{\mathrm{ox}}=2.3 \mathrm{GPa}$, which corresponds to a stiffness of the oxidation layer nearly 1000 times higher than the PDMS bulk one ${ }^{30}$.

The agreement between computational analysis and experiment gives the possibility to evaluate the shape of the squeezed nanochannel, which is not directly deducible from conductance measurements. In particular, simulations show that the deformation of our triangular nanochannel begins with the drop of its roof $(\mathrm{D} \leq 0.3)$ and, when the compression is increased, also its base undergoes a reduction. As an example, the simulated profile of the nanochannel for $\mathrm{D}=0.3$ is shown in figure $2 \mathrm{c}$. The bulk PDMS layer and the oxidation layer domains are represented in light and dark grey, respectively. The undeformed channel shape is indicated by a black line.

DNA manipulation. In order to study how nanochannel squeezing influences molecular translocation, we performed $\lambda$-DNA detection experiments at different compressive strain.

$\lambda$-DNA was added in the microchannel connected to the negative electrode. Once a positive voltage bias was applied, the negatively charged DNA molecules moved towards the positive electrode passing through the nanochannel, and translocation events were detected as transient current drops. Figure $3 \mathrm{a}-\mathrm{b}-\mathrm{c}$ reports three current traces recorded for compressive strain of $0,0.30$ and 0.35 , respectively, and by applying a voltage bias of $200 \mathrm{mV}$ (no software modifications have been performed). Several signal spikes appeared, corresponding to single molecule translocations, not present in the trace recorded by filling the device with the $\mathrm{KCl}$ buffer without DNA. During the nanochannel squeezing, the shape of the detected signal changed, as shown in figures $3 \mathrm{~d}-\mathrm{e}-\mathrm{f}$, for $\mathrm{D}=0,0.30$ and 0.35 , respectively. Considering that the noise band (about $20 \mathrm{pA}$ ) is half the mean event amplitude (about $40 \mathrm{pA}$ ), single events are well distinguishable 
a)

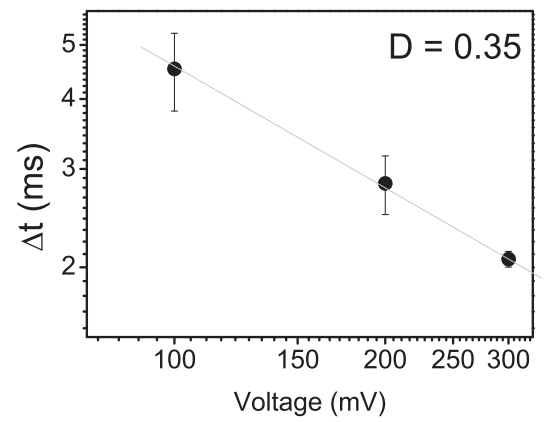

b)

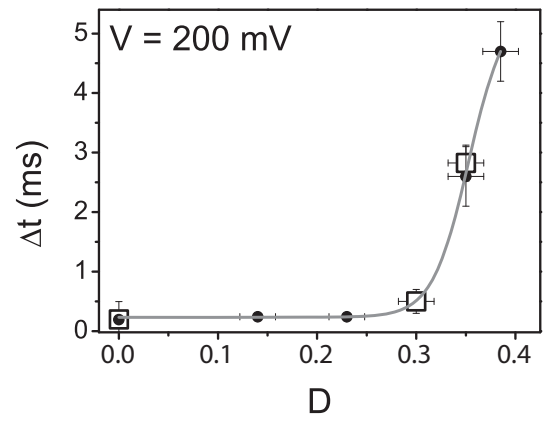

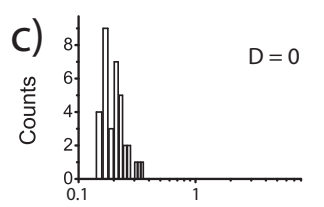
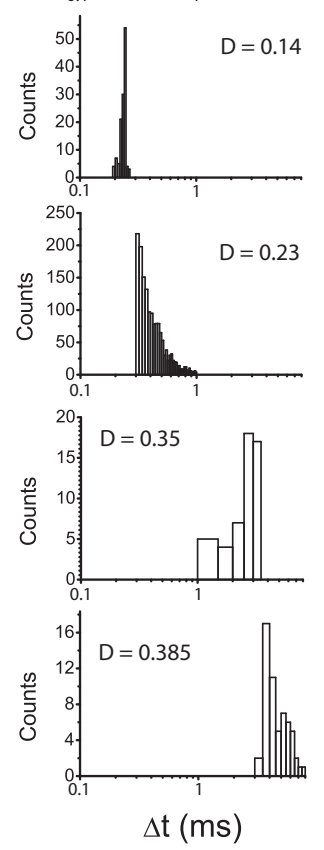

Figure $4 \mid$ DNA translocation data analysis. (a) Plot of event duration $(\Delta \mathrm{t})$ versus the applied voltage at $\mathrm{D}=0.35$. (b) Plot of event duration $\Delta \mathrm{t}$ versus the compressive strain $\mathrm{D}$ at $200 \mathrm{mV}$ applied voltage for two devices (circles and squares), $2 \mathrm{~h}$ acquisition time each. (c) Histograms of the event duration $\Delta \mathrm{t}$ at different compressive strain values $(\mathrm{D}=0,0.14,0.23,0.35$, $0.385)$ at $200 \mathrm{mV}$ applied voltage. Histograms are related to circular data points of figure $4 \mathrm{~b}$. The number of events are 35, 128, 1578, 59, 215 for respectively $\mathrm{D}=0, \mathrm{D}=0.14, \mathrm{D}=0.23, \mathrm{D}=0.35$ and $\mathrm{D}=0.385$.

and statistical analysis of current traces allows to identify them and to calculate their duration in order to deduce the mean translocation time, $\Delta \mathrm{t}$. In particular, to calculate the spike duration, the starting point of the event was set as the first point before the current trace falls below a fixed threshold, and the ending point as the first point after the current rises back above the threshold. Figures 4 a shows the plot of $\Delta t$ as a function of the applied voltage for $\mathrm{D}=0.35$ : when increasing the applied voltage $(\mathrm{V}), \Delta \mathrm{t}$ decreases, due to the enhanced molecule velocity, following the relation:

$$
\Delta \mathrm{t} \propto \mathrm{V}^{\beta}
$$

A fit was performed, obtaining $\beta=(-0.7 \pm 0.1)$. This behavior is expected for current fluctuations associated to translocation events of long DNA chain in nanochannels ${ }^{31-33}$.

Figure $4 \mathrm{~b}$ shows the plot of mean translocation time $\Delta \mathrm{t}(200 \mathrm{mV}$ of voltage bias) as a function of the compressive strain $\mathrm{D}$ for two different devices (circles and squares). Histograms (related to circular data points in figure $4 \mathrm{~b}$ ) of the event duration $\Delta \mathrm{t}$ at different compressive strain values $(\mathrm{D}=0,0.14,0.23,0.35,0.385)$ are shown in figure $4 c$.

By reducing the nanochannel dimensions (from an estimated section of $S_{0} \approx 35600 \mathrm{~nm}^{2}$ at $\mathrm{D}=0$ to $\mathrm{S}_{0.385} \approx 6800 \mathrm{~nm}^{2}$ at $\mathrm{D}=0.385$ ), the duration of translocation events increases.

\section{Discussion}

In this study we describe the fabrication and use of an elastomeric nanochannel device. In particular, our results demonstrate the possibility of tuning the size of the nanochannel - thus controlling its cross section - in order to alter the translocation dynamics of $\lambda$ DNA molecules. The device characterization has been performed both experimentally and numerically. The squeezing experiment demonstrates that we are able to control deformations at the nanoscale by simply applying an external macroscopic compressive strain. The smallest section that we experimentally obtained during squeezing experiments was $\mathrm{S}=68 \mathrm{~nm}^{2}$, corresponding to $\mathrm{D}=0.55$.

Simulations were performed in order to understand how the nanochannel is deformed by the applied compression, up to a strain value of $\mathrm{D}=0.35$. The nanochannel squeezing strongly depends on the mechanical properties of the device, such as the Young's modulus of the oxidation layer. A good agreement between experiments and simulations was achieved by using a value for the stiffness of the oxidation layer that is three orders of magnitude higher than that of the bulk PDMS. After setting this value, we performed simulations to predict the channel shape (figure $2 \mathrm{c}$ ). In particular, this analysis revealed that the nanochannel aspect ratio is high for all the values of compressive strain $(\mathrm{b} / \mathrm{h} \gg 1)$. This information is crucial to understand the confinement capabilities of the nanochannel. Indeed, the DNA manipulation experiment demonstrates that the confinement of DNA molecules during their translocation through the nanochannel strongly depends on the applied compressive strain (D), i.e. on the nanochannel deformation. In particular, it is possible to observe a sharp increase in the translocation time at $\mathrm{D}=0.3$ (figure $4 \mathrm{~b}$ ). For instance, the translocation time at $\mathrm{D}=0.385$ is one order of magnitude higher than the one obtained at $\mathrm{D}=0$.

This trend can be explained by considering the conformational changes that a DNA molecule experiences when it is forced to enter and pass through the nanochannel. In fact, when no compression is imposed on the device, DNA chains acquire a blob conformation inside the nanochannel, as predicted by the De Gennes' model ${ }^{34}$. Conversely, when a high compression is imposed on the top of the device, the channel cross section is subjected to a strong reduction. Under this confinement condition, $\lambda$-DNA molecules undergo a conformational change as described by Odijk ${ }^{35}$. For triangular nanochannels, these scaling laws cannot be directly applied in order to obtain the correct value for the DNA molecule elongation at different applied displacements. Nevertheless, we speculate that conformational changes, due to the unwinding process at the entrance of the nanochannel, affect the translocation time: when entering channels with smaller cross sections, a DNA molecule needs more time to unwind and to pass through it, i.e. the translocation time increases.

Due to the complexity of the process and the particular shape of these nanostructures, a direct comparison between the results reported in figure $4 \mathrm{~b}$ and data from other systems is not possible. Indeed, the DNA confinement and translocation dynamics in our nanochannel are different with respect to typical solid state or biological nanopores due to the huge length of our channel $-15 \mu \mathrm{m}$ compared to 5-100 $\mathrm{nm}$ long nanopores - and to the different shape of the cross section (triangular versus circular). However, despite differences, Fan et al. ${ }^{36}$ demonstrated that the translocation time of $\lambda$-DNA in a $10 \mu \mathrm{m}$ long nanotube (50nm diameter) ranges from 4 to $10 \mathrm{~ms}$, a value which is in good agreement with our result at high $\mathrm{D}$.

In conclusion, we demonstrated that it is possible to control and reversibly tune the dimension of a nanochannel fabricated using elastomeric materials, so to fit the target molecule dimensions. We electrically detected single DNA molecules at different squeezing conditions, demonstrating the possibility to manipulate the DNA passage inside the nanochannel: the translocation time obtained at high applied displacement is an order of magnitude higher compared to the one obtained without applying compression. The possibility to dynamically control the nanochannel dimension opens up new opportunities to study the interactions between biomolecules and nanochannels, such as the dependence of the translocation dynamics on the channel size, and effects of moving walls ${ }^{37}$. On the other hand, 
nanochannel manipulation offers great opportunities in biosensing, especially for single molecule analysis, and it is promising for further development in ultra rapid sequencing technology, which would greatly benefit from a significant slow down of DNA molecules.

\section{Methods}

Fabrication procedure. A silicon substrate was micromachined by standard lithographic techniques creating two microchannels (height $50 \mu \mathrm{m}$, width $500 \mu \mathrm{m}$ ) and four reservoirs. The distance between the two microchannels in the central zone is $200 \mu \mathrm{m}$. Cylindrical pillars ( $50 \mu \mathrm{m}$ height and $50 \mu \mathrm{m}$ diameter) were arranged along the microchannels to avoid polymeric replica collapse. A CrossBeam@ workstation $1540 \mathrm{XB}$ model (Zeiss) that combines an ultra-high resolution scanning electron microscope (UHR-SEM) with a Focused Ion Beam (FIB) was then used to fabricate two facing trapezoidal excavations $(114 \mu \mathrm{m}$ larger base, $6.5 \mu \mathrm{m}$ smaller base, $92.5 \mu \mathrm{m}$ height, and the depth flagged gradually from $20 \mu \mathrm{m}$ to $2 \mu \mathrm{m}$ ) to reduce the distance between the two microchannels down to $15 \mu \mathrm{m}$ and to create the nanochannel.

Afterwards, poly(dimethylsiloxane) (PDMS) was used to replicate the master: the final positive replica was composed by a thin PDMS 3:1 layer (prepolymer and curing agent ratio 3:1) supported by a thick PDMS 10:1 layer.

The fabrication started with the exposition of the silicon master to $1 \mathrm{H}, 1 \mathrm{H}, 2 \mathrm{H}, 2 \mathrm{H}$ per-FluoroOctylTrichloroSilane (FOTS) to create an anti-stiction layer. The negative replica was fabricated by spin coating PDMS 3:1 on the master. After 30 minutes baking $\left(60^{\circ} \mathrm{C}\right)$, PDMS 10:1 was deposited on the top of the master, resulting in a thick (about $5 \mathrm{~mm}$ ) layer. After 4 hours baking $\left(60^{\circ} \mathrm{C}\right)$, the negative replica was peeled off. Then the negative replica was exposed to $1 \mathrm{H}, 1 \mathrm{H}, 2 \mathrm{H}, 2 \mathrm{H}$-per-

FluoroOctylTrichloroSilane (FOTS) in order to create an anti-stiction layer. The positive replica was produced from the negative replica following the same procedure: spin coating PDMS 3:1, 30 min baking, spin coating 10:1, 4 hours baking and peel off.

In order to obtain an enclosed nanochannel, the elastomeric device and a glass cover slip were treated for 60 s in oxygen plasma with a power of $30 \mathrm{~W}$ and put into conformal contact to achieve an irreversible bond.

The device was then connected to an external fluidic apparatus and placed in a double Faraday cage enclosure on an anti-vibration table.

Experimental details. Conductance measurements were performed filling the device with $100 \mu \mathrm{l}$ of a $\mathrm{KCl} 1 \mathrm{M}$ solution. Two $\mathrm{Ag} / \mathrm{AgCl}$ electrodes allowed to apply the voltage and to collect the ionic current by means of a patch-clamp amplifier (Axopatch 200B, Axon Instruments). The sampling rate for ionic current measurement was set at $250 \mathrm{kHz}$ and the internal low-pass four pole Bessel filter was set at $10 \mathrm{kHz}$.

For the DNA detection experiment, $\lambda$-DNA (48.5 kbp WAKO) was diluted in $\mathrm{KCl}$ $1 \mathrm{M}\left(3 \mathrm{ng} \mu \mathrm{l}^{-1}\right)$.

Simulations. We performed plane-strain hyperelastic finite element analysis imposing a displacement, $\Delta \mathrm{L}$, on the top of the device and analyzing the closure of the nanochannel ${ }^{30}$ in order to simulate experimental conditions. We evaluated the squeezing of a triangular cross section nanochannel with height $\mathrm{h}$ and base $\mathrm{b}$, obtained from AFM images: $\mathrm{h}=(70 \pm 5) \mathrm{nm}, \mathrm{b}=(1.0 \pm 0.3) \mu \mathrm{m}$. Considering that PDMS 3:1 and PDMS 10:1 have similar initial shear modulus, we modeled the device as a bilayer composed by a PDMS 10:1 layer and an oxidized PDMS layer at the interface between the PDMS replica and the glass cover slip. The oxidation layer was considered as a $100 \mathrm{~nm}$ thick layer of elastic material with Young's modulus $\mathrm{E}_{\mathrm{ox}}=$ $2.3 \mathrm{GPa}$. The initial shear modulus and the initial bulk modulus of bulk PDMS were set equal to $\mu=0.77 \mathrm{GPa}$ and $\mathrm{K}=7.7 \mathrm{GPa}$ respectively. The covalent bonding between the polymeric surface and the glass cover slip was simulated using a fixed boundary condition. The contribution of the work of adhesion between glass and PDMS was neglected. A varying displacement was applied on the top of the device and, for every value of the compressive strain $\mathrm{D}$, the open section of the squeezed nanochannel was evaluated and related to its conductance by using the Ohm's law.

1. Mark, D., Haeberle, S., Roth, G., Von Stetten, F. \& Zengerle, R. Microfluidic labon-a-chip platforms: requirements, characteristics and applications. Chem. Soc. Rev. 39, 1153-1182 (2010).

2. Kasianowicz, J. J., Brandin, E., Branton, D. \& Deamer, D. W. Characterization of Individual Polynucleotide Molecules Using a Membrane Channel. Proc. Nat. Ac. Sci. 93, 13770 (1996).

3. Keyser, U. F. Controlling Molecular Transport through Nanopores. J. R. Soc. Interface 8, 1369 (2011).

4. Kowalczyk, S. W., Tuijtel, M. W., Donkers, S. P. \& Dekker, C. Unraveling single-stranded DNA in a solid-state nanopore. Nano Lett. 10, 1414-1420 (2010).

5. Li, J., Gershow, M., Stein, D., Brandin, E. \& Golovchenko, J. A. DNA molecules and configurations in a solid-state nanopore microscope. Nat. Mater. 2, 611-615 (2003).

6. Keyser, U. F. et al., C. Direct Force measurements on DNA in a solid-state nanopore. Nature Physics 2, 473-477 (2006).

7. Matysiak, S. et al. Dynamics of Polymer Translocation through Nanopores: Theory Meets Experiment. Phys. Rev. Lett. 96, 118103 (2006).

8. Fologea, D., Uplinger, J., Thomas, B., McNabb, D. S. \& Li, J. Slowing DNA Translocation in a Solid-State Nanopore. Nano Lett 5, 1734 (2005).
9. Brun, L. et al. Dynamics of Polyelectrolyte Transport through a Protein Channel as a Function of Applied Voltage. Phys. Rev. Lett. 100, 158302 (2008).

10. A.Meller, A., Nivon, L. \& Branton, D. Voltage-Driven DNA Translocations through a Nanopore. Phys. Rev. Lett. 86, 3435 (2001).

11. Rincon-Restrepo, M., Mikhailova, E., Bayley, H. \& Maglia, G. Controlled Translocation of individual DNA molecules through protein nanopores with engineered molecular brakes. Nano Lett. 11, 746-750 (2011).

12. Keyser, U. F., Van der Does, J. Dekker, C. \& Dekker, N. H. Optical Tweezers for Force Measurements on DNA in Nanopores. Rev. Sci. Instrum. 77, 105105 (2006)

13. Trepagnier, E. H., Radenovic, A., Sivak, D., Geissler, P. \& Liphardt, J. Controlling DNA capture and propagation through artificial nanopores. Nano Lett. 7, 2824-2830 (2007).

14. Peng, H. \& Ling, X. S. Reverse DNA translocation through a solid-state nanopore by magnetic tweezers. Nanotechnology 20, 185101 (2009).

15. Siwy, Z. S. \& Howorka, S. Engineered Voltage-Responsive Nanopores. Chem. Soc. Rev. 39, 1115 (2010).

16. Yameen, B., Ali, M., Neumann, R., Ensinger, W., Knoll, W. \& Azzaroni, O. Ionic Transport Through Single Solid-State Nanopores Controlled with Thermally Nanoactuated Macromolecular Gates. Small 5, 1287 (2009).

17. Kowalczyk, S. W., Blosser, T. R. \& Dekker, C. Biomimetic nanopores: learning from and about nature. Trends in Biotechnology, 29, 607-614 (2011).

18. Roberts, G. S., Kozak, D., Anderson, W., Broom, M. F., Vogel, R. \& Trau, M. Tunable Nano/Micropores for particle detection and discrimination: scanning ion occlusion spectroscopy. Small 6, 2653-2658 (2010).

19. Vogel, R. et al. Quantitative sizing of nano/microparticles with a tunable elastomeric pore sensor. Anal. Chem. 83, 3499-3506 (2011).

20. Sowerby, S. J. \& Petersen, G. B. A proposition for single molecule DNA sequencing through a nanopore entropic trap. Int. J. Nanothchnol. 6, 398-407 (2009).

21. Sowerby, S. J., Broom, M. F. \& Petersen, G. B. Dynamically resizable nanometerscale apertures for molecular sensing. Sensor and Actuators B 123, 325-330 (2007).

22. Huh, D. et al. Tuneable Elastomeric Nanochannels for Nanofluidic Manipulation. Nature Materials 6, 424 (2007).

23. Angeli, E., Manneschi, C., Repetto, L., Firpo, G. \& Valbusa, U. DNA Manipulation with Elastomeric Nanostructures Fabricated by Soft-Moulding of a FIB-Patterned Stamp. Lab Chip 11, 2625 (2011).

24. Fanzio, P. et al. DNA Detection with a Polymeric Nanochannel Device. Lab Chip 11, 2961 (2011).

25. Lee, J. N., Park, C. \& Whitesides, G. M. Solvent compatibility of Poly(dimethylsiloxane)-based microfluidic devices. Anal. Chem. 75, 6544-6554 (2003).

26. Mills, K. L., Zhu, X., Takayama, S. \& Thouless, M. D. The Mechanical Properties of a Surface-Modified Layer on Polydimethylsiloxane. J. Mater. Res. 23, 37 (2008).

27. Mussi, V. et al. DNA-Functionalized Solid State Nanopore for Biosensing. Nanotechnology 21, 145102 (2010).

28. Mussi, V. et al. Electrical Characterization of DNA-Functionalized Solid State Nanopores for Bio-Sensing. J. Phys.: Condens. Matter 22, 454104 (2010).

29. Kowalczyk, S. W., Grosberg, A. Y., Rabin, Y. \& Dekker, C. Modeling the conductance and DNA blockade of solid-state nanopores. Nanotechnology. 22, 315101 (2011).

30. Manneschi, C. et al. Mechanical squeezing of an elastomeric nanochannel device. Microfluid. Nanofluid. DOI: 10.1007/s10404-012-1018-3

31. Fologea, D. et al. Detecting Single Stranded DNA with a Solid State Nanopore. Nano Lett 5, 1905 (2005)

32. Saito, T. \& Sakaue, T. Dynamical Diagram and Scaling in Polymer Driven Translocation. The European Physical Journal E: Soft Matter and Biological Physics 34, 135 (2011).

33. Yong, H., Wang, Y., Yuan, S., Xu, B. \& Luo, K. Driven polymer translocation through a cylindrical nanochannel: interplay between the channel length and the chain length. Soft Matter 8, 2769 (2012).

34. De Gennes, P. G. Scaling concepts in polymer physics. (Cornell Univ Pr: 1979).

35. Odijk, T. The Statistics and Dynamics of Confined or Entangled Stiff Polymers. Macromolecules 16, 1340 (1983).

36. Fan, R., Karnik, R., Yue, M., Li, D., Majumdar, A. \& Yang, P. DNA translocation in inorganic nanotubes. Nano Lett. 5, 1633-1637 (2005).

37. Cohen, J. A., Chaudhuri, A. \& Golestanian R. Active Polymer Translocation through Flickering Pores. Phys. Rev. Lett., 107, 238102 (2011).

\section{Acknowledgments}

This work has been supported by Ministero dell'Università e della Ricerca (MIUR), Italy, with the National Project NANOMAX.

\section{Author contributions}

P. Fanzio, V. Mussi and E. Angeli designed the experiment. G. Firpo and L. Repetto patterned the silicon master. L. Ceseracciu studied the elastic properties of PDMS. P. Fanzio fabricated the polymeric device and performed ionic current measurements for the squeezing experiment and for the DNA detection experiment. C. Manneschi simulated the device deformation. P. Fanzio, C. Manneschi and U. Valbusa wrote the paper. 


\section{Additional information}

Competing financial interests: The authors declare no competing financial interests

License: This work is licensed under a Creative Commons

Attribution-NonCommercial-NoDerivative Works 3.0 Unported License. To view a copy

of this license, visit http://creativecommons.org/licenses/by-nc-nd/3.0/
How to cite this article: Fanzio, P. et al. Modulating Dna Translocation by a Controlled Deformation of a Pdms Nanochannel Device. Sci. Rep. 2, 791; DOI:10.1038/srep00791 (2012). 